\title{
SENSORY FEEDBACK MYOELECTRIC ARM
}

\section{CHANDRA SEKHAR ASHOK ${ }^{1}$, SWAROOPA SHIGLI ${ }^{2}$, PANDURANG MIRAJKAR ${ }^{3}$, Dr. N. BHOOPAL ${ }^{4} \&$ PROF. ASHOK SHIGLI ${ }^{5}$}

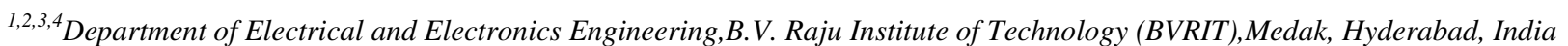

${ }^{5}$ Department of Bio Medical Engineering,B.V. Raju Institute of Technology (BVRIT),Medak, Hyderabad, India

\begin{abstract}
The demand for prosthetics in the market with autonomous and automatic controlling of finger movements using embedded systems is exponential. However this autonomy accompanies heavy usage of components for perfection resulting in costs becoming uncompromised for such a device. Practically tested results show that these need-based people have active upper arm functioning, which serves as an advantage for using the electromyography (EMG) and sensory feedback to develop the prosthetic lower arm. EMG for a subject is always challenging in case of precise placement of electrodes at various muscle points to measure the muscle activity through a surgery. Therefore the belowproposed model is developed carefully to avoid any further operating procedures along with added features such as sensory feedback for temperature and force-touch sensors including a withdrawl-mechanism within the prosthetic arm.

KEYWORDS: Partial arm amputation, human arm anatomy, Pressure-based myoelectric control, Lower arm prosthetics, sensory feedback, Temperature sensor, force-touch (pressure) sensor \& Electromyography
\end{abstract}

Received: Jun 08, 2020; Accepted: Jun 28, 2020; Published: Sep 03, 2020; Paper Id.: IJMPERDJUN20201046

\section{INTRODUCTION}

The sensory feedback myoelectric arm, as the name implies, uses myoelectric signals (EMG) for control and operation. The neuro-muscular system in the upper limb of the patients persists unharmed which acts as a source for obtaining active muscular movements and nerve senses which is suitable to develop an artificial arm accordingly after careful examination and processing. The arm is made up of composite silica-gel material and comprises human-like traits, namely temperature sensing for safety and pressure sensing for grasping of various kinds of objects.

\section{DESIGN AND DEVELOPMENT}

The human anatomy of the hand is depicted in Figure 1.

Prosthetic size, weight, and autonomy are the main design demands which play a vital role in its usage. Any disturbances with these specifications may result in impractical workability and usage of the prosthetic hand, and hence a decline may occur in the product's usage. Therefore these specifications are to be addressed carefully to avert unfeasible design issues and should be replaceable for additional improvements in the near future once its present physical implementation is in order. 


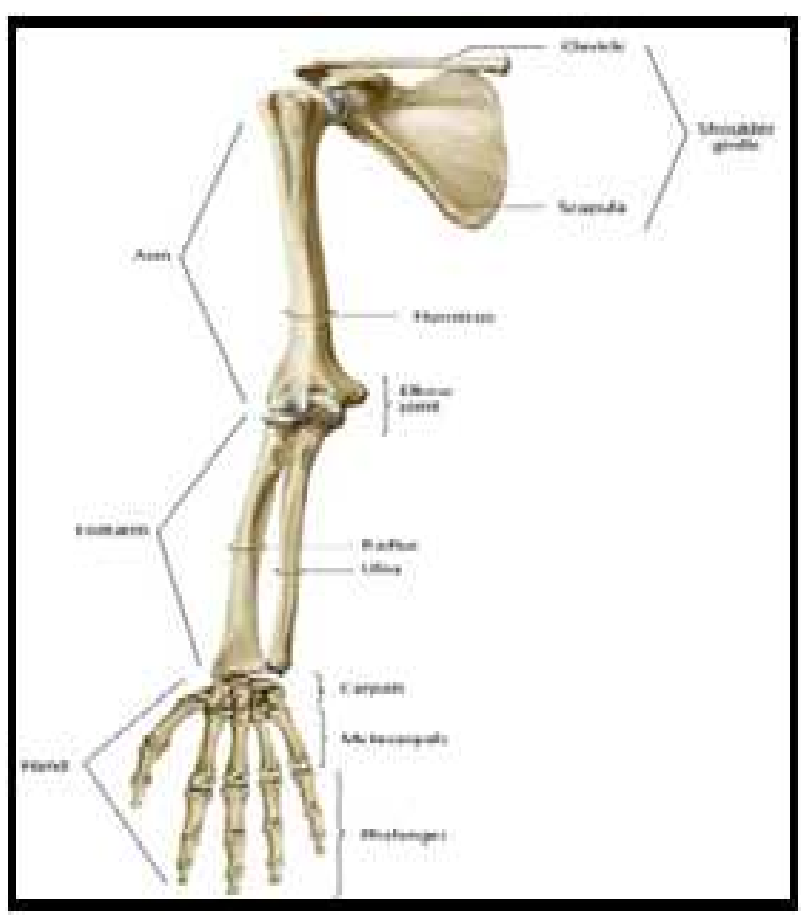

Figure 1: Anterior view of Full Arm and Hand

The average weight of a human hand is about $400 \mathrm{~g}$. In comparison, the prosthetic hand weight considerably varies in between 350 to $615 \mathrm{~g}$ and 350 to 2,200 $\mathrm{g}$ in commercial and research-based hands, respectively [1]. This weight is typically concluded as very heavy by the users because, in contrast to the natural human hand, prosthetic hand applies force on the muscle at the implant area instead of the skeleton. This can cause discomfort to the user with increasing usage hours. This project aims to develop such a design which would be as light as possible by replacing the sophisticated electronics and mechanical devices with lighter yet effective ones.

The complexities within the human hand due to an exhibition of 27 degrees of freedom, make it difficult for an artificial arm to resemble all the features for the flawless functioning of the whole arm. On the contrary, an extent of these attributes can be instilled into an artificial arm provided the use of the number of components is much higher with high-end electronics and mechanical devices which in turn blows-up the cost of the artificial arm. These arms are which we find in the market today.

This project presents sensory feedback based prosthetic arm, which has been approved by the test subjects as comfortable and moderately weighted, yet very effective for its model. All the components utilized and the material used for the sheathing is substantially cost-effective and easily replaceable with the model containing a sufficient amount of space for adjustability for the user. The precision grip only is accommodated due to the fact that almost all the daily activities (ADLs) involve this type of gripping. The Silicone material is used to make a real looking arm by taking the measurements of the other arm and colouring according to the skin tone of the user.

\section{WHY SENSORY FEEDBACK?}

An essential feature of the bionic hand design is sensory feedback. It is the base for the operation of the arm; which almost all the time has precise feedback through its components used, hence a very human-like operation. By the inclusion of force sensor, the ability to measure the applied force on each finger, and then be able to react to that force, leads to better 
user experience, making the user capable of grasping a fragile object without breaking them, and allowing the microcontroller to stop the motors before stalling them and burning out.[2]

Another addition to the prosthesis to make it much more realistic is by including the temperature sensor/thermistors within the feedback system. This way the fingertips of the arm can sense the temperature and the feedback system which consists of a Peltier/cooler module which in turn conveys the temperature to the user by cooling or heating up accordingly through the ceramic plates with a ceramic semiconductor between them. This module conducts the temperature from the top to bottom surface by flowing current, hence giving the approximate sense of the cold or heat nature of the object.

\section{A. Withdrawal-Mechanism}

The feedback mechanism in our prosthesis arm design includes an added feature, the withdrawal-mechanism. If the hand comes in contact with an object whose temperature differs from the pre-set values at the fingertips, it results in the withdrawal of the fingers naturally.

Finally, a vibrator module is integrated into the prosthesis arm which vibrates according to the force utilized by the hand for gripping, in other words when there is a change in the gripping force due to pressure on the hand. This informs the user that the signal coming from the muscles while trying to hold an object by flexing them. The signal is being read and analyzed by the microcontroller, as a result of which the action is being performed as expected. The coin-vibrators' output varies in accordance to the pressure applied/received. The EMG processes this signal from muscle areas to apply as much pressure required to do the activity. The EMG signal is amplified by an op-amp and sent to the microcontroller for more complete precise action.

\section{COMPONENTS \& THEIR SPECIFICATIONS}

Components were chosen in such a way so as to reduce manufacturer complexity and to put the replacing ability to a much simpler extent. For good performance in daily activities, torque and speed of these components were considered to be essential and were made adequate using these components.

The objective of 1.5 to 2 second hand-closing time is pre-set. The EC2SA-05D05 DC Micromotor with input nominal voltage of $5 \mathrm{~V}$, output nominal voltage of $+/-5 \mathrm{~V}$, and efficiency of $77 \%$ is used. The L298N driver module, using ST's L298N chip, is used to drive the DC Motor, which can easily control the DC motor speed and direction. REES52 Esp32 board is the microcontroller for the project which effectively drives every function of the myoelectric arm. This board is small, effective and Arduino compatible and gives an update to the arm.

Peltier Cooler or Cooling Module, a generic 12706 Thermoelectric of 12V 92W Max which creates a temperature differential on each side is mounted on the active part of the upper arm. It is designed for cooling and heating up to 90 degrees Celsius for its applications.

CHPSS515 Ntc 100K Ohm 3950 Thermistor with highly advantageous features of high accuracy and small size along with a small Keywholesale Force Sensor of 5.08mm Circle and 0.16" (4 mm) diameter active sensing area with inversely varying resistance with pressure are mounted at the sensing areas, i.e. the tips of the fingers.

Lastly, an in-house sensory vibrator/ electromyography model is developed with the two coin vibrator motors with a size of $10 \mathrm{~mm}$ and a diameter of $2.8 \mathrm{~mm}$, rated voltage, and current being $1 \mathrm{~V}$ to $4.2 \mathrm{~V}$ and $66 \mathrm{~mA}$ respectively. It has 
an output speed of $12000 \mathrm{rpm}$. The model is developed, keeping all necessary features in mind, which, along with an amplifier, records the healthy nerve cells' functions to help operate the arm desirably. The source of power is a $10 \mathrm{~V}$ battery which demonstrates enough for the arm to operate efficiently.

\section{COMPONENTS' PLACEMENT}

The functioning of the arm begins when either or all of the sensors, namely, force sensor, temperature sensor, and EMG are triggered according to the action performed by the user's arm.

The action taken by the user making use of the EMG or temperature sensor sets off the stepper motor through the driver module, which regulates the flow of voltages across the arm. The temperature sensor is connected to the controller, which modulates the signal through PWM (pulse width modulation) in the RC-circuit provided and regulates the power to pass to the Peltier module.

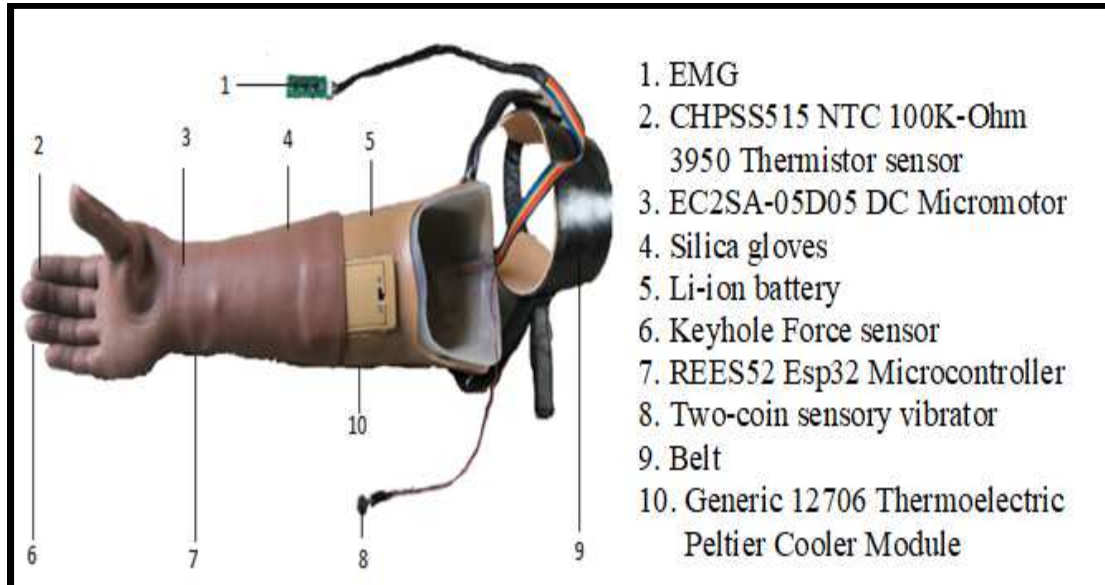

Figure 2:Sensory Feedback Myoelectric Arms

This temperature is sensed by the exposed skin underneath the Peltier module.The force sensor activates the coin vibrator module, i.e. as the pressure by or on the hand increases, the corresponding voltage reflects on the vibrator giving a sense of the pressure exerted. The signal can be feeble; hence an operational amplifier-based RC-filter design is connected to strengthen the signal to be processed by the microcontroller. 


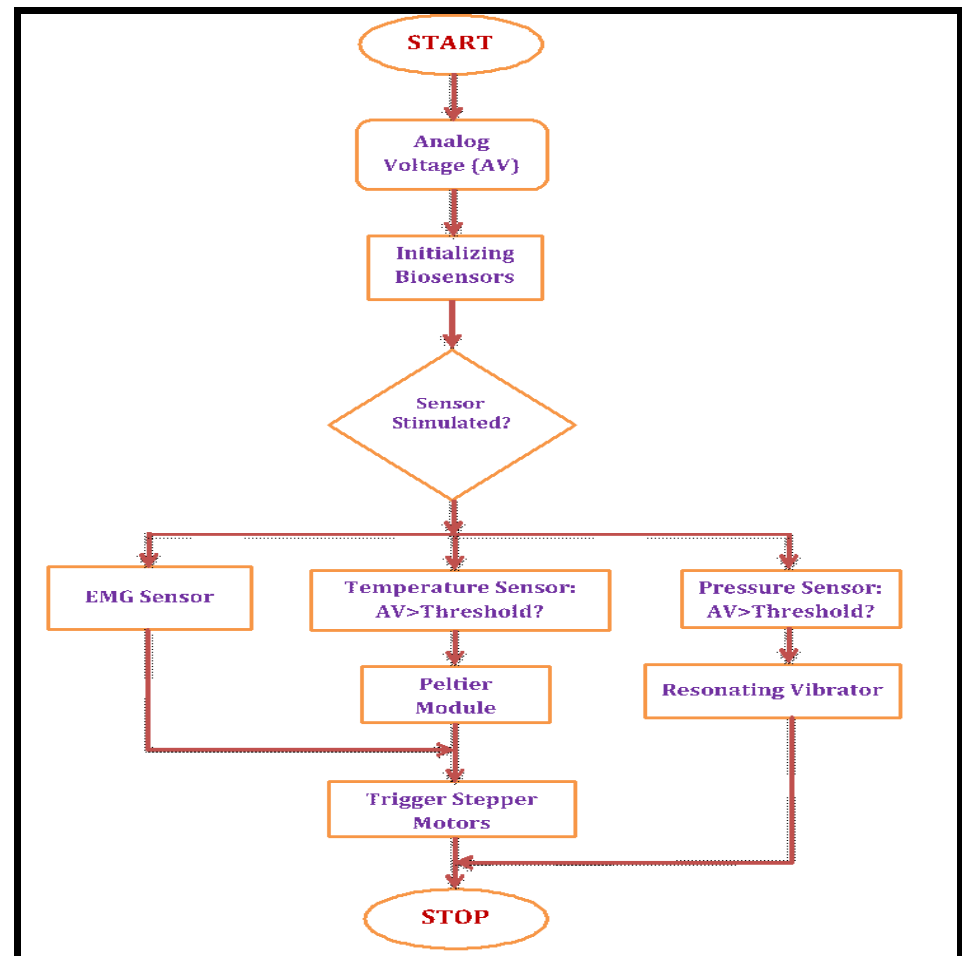

Figure 3: Flow Chart

\section{DEVELOPING THE EMG SENSORY VIBRATOR MODULE}

The Electromyograph (EMG) is the remarkable achievement of this myoelectric prosthetic arm. EMG is a medical electrodiagnostic sensor which will generate the electrical signal proportionally to the skeletal muscle movement or vibrations. So far the output signal of the designed EMG has got more harmonics which results in more total harmonic distortion (THD). As we progressed towards mitigating the problems in performance, we came out with an operational amplifier-based RC-filter design as follows:

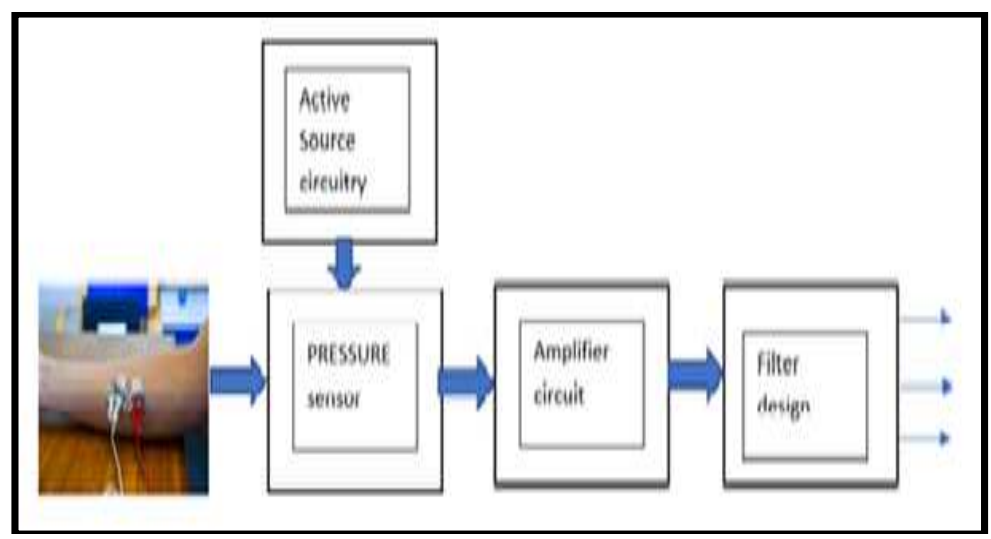

Figure 4: Op-amp based RC-filter design

\section{A. Active Source Circuitry}

The input voltage $12 \mathrm{v}$ supplied to DC-DC converter (EC2SA-05D05) is interfaced in the circuitry to get the availability of $+/-5 \mathrm{v}$. this design enhances the mobilization of the prosthetic arm so that people can use the designed arm as their daily essentials. 
Table 1: Active Source Circuitry Specifications

\begin{tabular}{|l|l|l|l|l|l|l|l|}
\hline $\begin{array}{l}\text { I/P } \\
\text { Volt }\end{array}$ & $\begin{array}{l}\text { O/P } \\
\text { Volt. }\end{array}$ & $\begin{array}{l}\text { Min. } \\
\text { O/P } \\
\text { I }\end{array}$ & $\begin{array}{l}\text { Max. } \\
\text { O/P } \\
\text { I }\end{array}$ & $\begin{array}{l}\text { I/P } \\
\text { I }\end{array}$ & $\begin{array}{l}\text { Full } \\
\text { Loa } \\
\text { d } \\
\text { I }\end{array}$ & $\begin{array}{l}\text { Load } \\
\mu \mathrm{F}\end{array}$ \\
\hline $\begin{array}{l}4.5 \mathrm{~V} \\
-9 \mathrm{~V}\end{array}$ & $\begin{array}{l}+/- \\
5 \mathrm{~V}\end{array}$ & $\begin{array}{l}0 \\
\mathrm{~mA}\end{array}$ & $\begin{array}{l}+/- \\
200 \mathrm{~m} \\
\mathrm{~A}\end{array}$ & $\begin{array}{l}60 \mathrm{~m} \\
\mathrm{~A}\end{array}$ & $\begin{array}{l}519 \\
\mathrm{~mA}\end{array}$ & 77 & $\begin{array}{l}200 \\
\mu \mathrm{F}\end{array}$ \\
\hline
\end{tabular}

Where,

$$
\text { Volt. = voltage }
$$

$\mathrm{I}=$ current

$\eta=$ efficiency

Load $\mu \mathrm{F}=$ load capacitance

As EMG is crucial in increasing the degrees of freedom as well as providing life-like mobility of the prosthetic arm, the focus is on precise and high sensitivity EMG design, hence this model is designed to obtain the expected outcome as follows:
A. Sensory feedback to the processor and motorized fingers
B. Accurate signal sensitivity, free from harmonics
C. Consistency in the critical threshold signal values.

\section{SIGNAL ACQUISITION FROM THE SENSORS}

To experiment with the prosthetic module, four test-subjects were appointed and the readings were recorded and compared along the developmental stages of the module.

\section{A. EMG Signal Acquisition}

To get the pinpoint areas of muscle excitation, EMG signal acquisition was performed by placing the differential electrodes using the Advancer Technologies EMG module hence throwing light on the muscle action potentials of various muscles.

The electrical activity of the muscle during nerves' stimulation was acquired with a digital oscilloscope. The muscles' action potentials were very minimal when the subjects were in a relaxed state and high for a high contraction of the muscles generating higher voltages. Thus it provided an appropriate choice of placing electrodes to get maximum muscle excitation.

The subjects were asked to put pressure on a soft material(foam) with high efforts for a while. The electrodes were placed at four different muscles to obtain their readings. The readings were frozen and the generated voltages in the corresponding time intervals were recorded. 


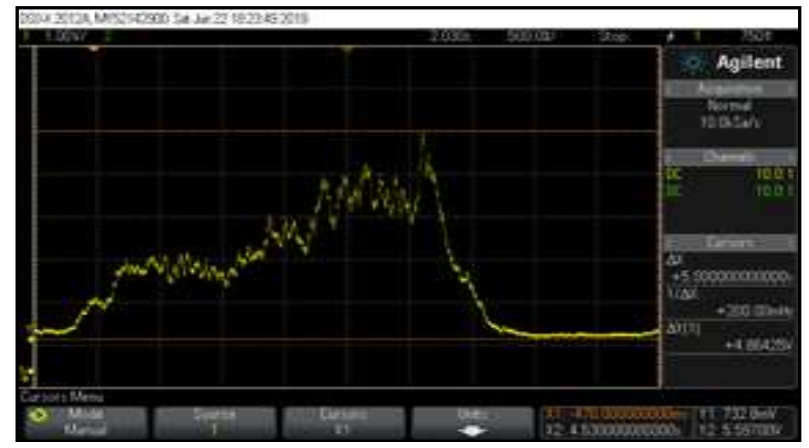

Figure 5: Electrodes placed at the Bicep muscles and their corresponding EMG values recorded on the CRO.

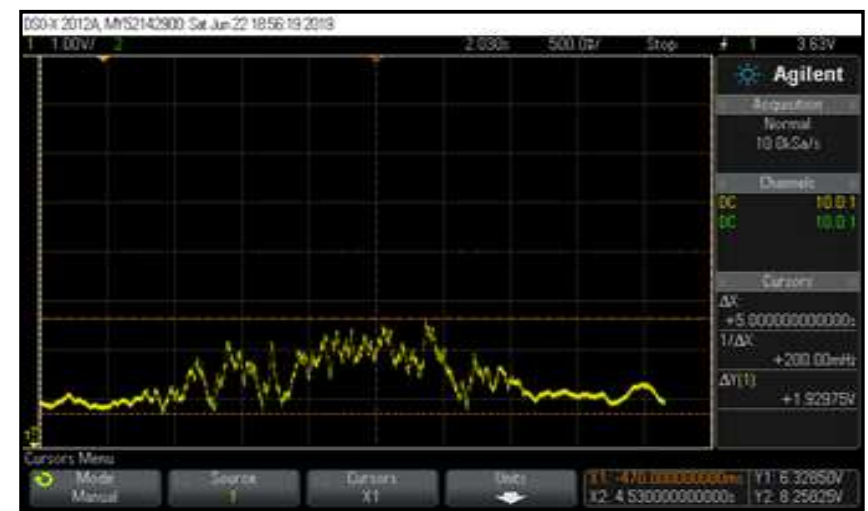

Figure 6 Electrodes placed at the Radius muscles and their corresponding EMG values recorded on the CRO.

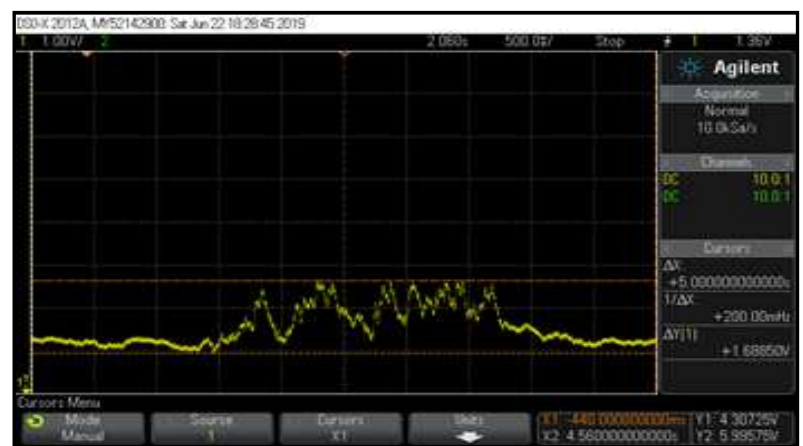

Figure 7:Electrodes placed at the Pronator-teres muscles and their corresponding EMG values recorded on the CRO.

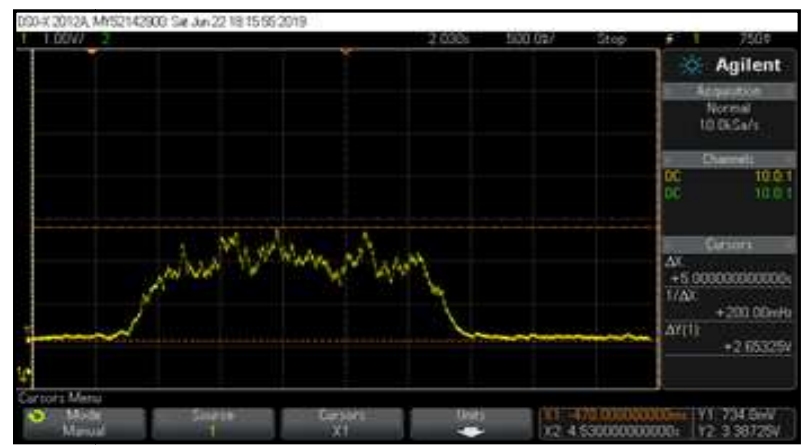

Figure 8: Electrodes placed at the Triceps muscles and their corresponding EMG values recorded on the CRO. 
Table 2:The Voltages Generated at Various Active Region Muscle Contractions

\begin{tabular}{|c|l|l|l|l|l|}
\hline S. No. & Electrodes Placement & Sub1 & Sub2 & Sub3 & Sub4 \\
\hline 1 & Biceps & 2.17 & 3.38 & 4.86 & 2.13 \\
\hline 2 & Radius & 1.61 & 2.17 & 1.92 & 1.69 \\
\hline 3 & Pronator Teres & 1.77 & 3.66 & 1.68 & 1.21 \\
\hline 4 & Tricep & 2.45 & 2.64 & 2.65 & 1.93 \\
\hline
\end{tabular}

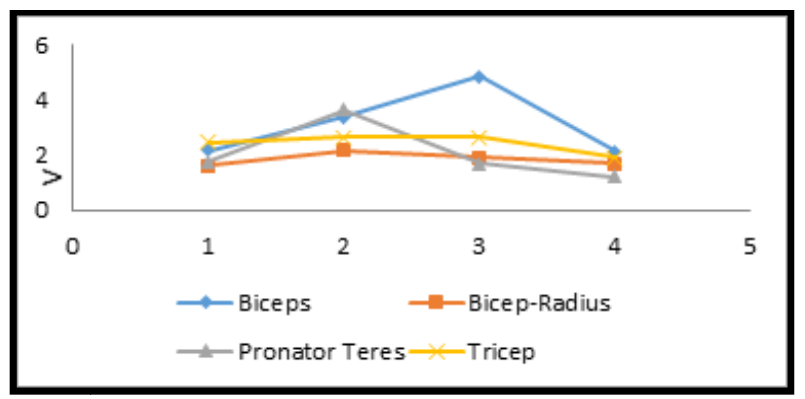

Figure 9: Graphical Representation of Muscle Contractions

From Table 2 we can observe that the highest EMG values were recorded for the Bicep muscles as expected and the weakest was Radius muscle.

\section{B. $\quad$ Force - Touch (Pressure) Sensor Signal Acquisition}

The touch sensors are placed on the tips of the fingers and accordingly, the pressure is increased to record the observation by each finger. The signals show the pressure applied by each of the fingers on the sensor. The signal strength varies for each of them with the first three i.e. thumb, index, and middle fingers recording the highest. Although the no. of subjects varied, the strength of the signals remained quite similar deriving the conclusion that the first three fingers are most essential or the functioning of the arm.

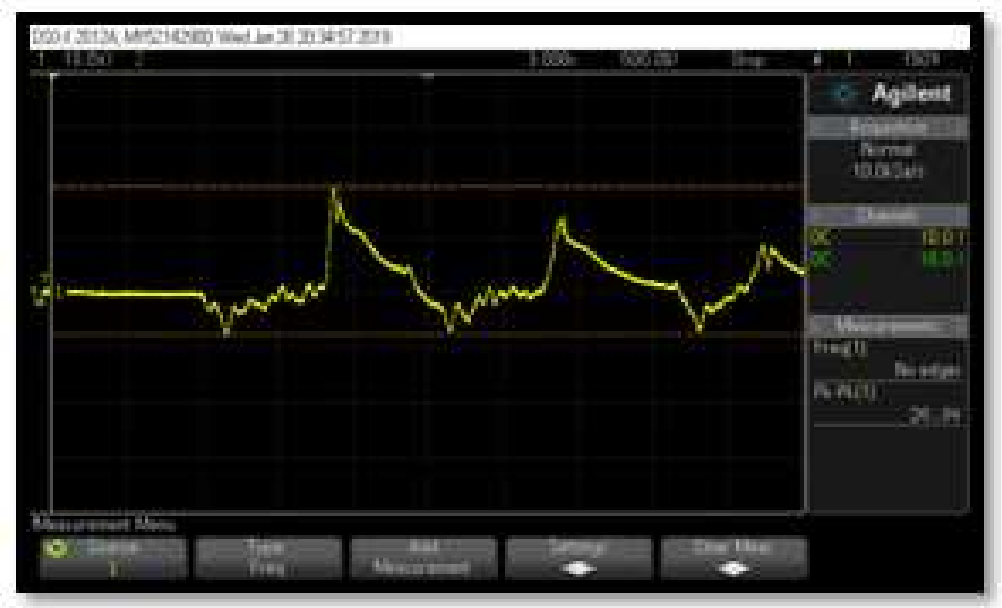

Figure 10:Thumb Finger Pressure Recording 


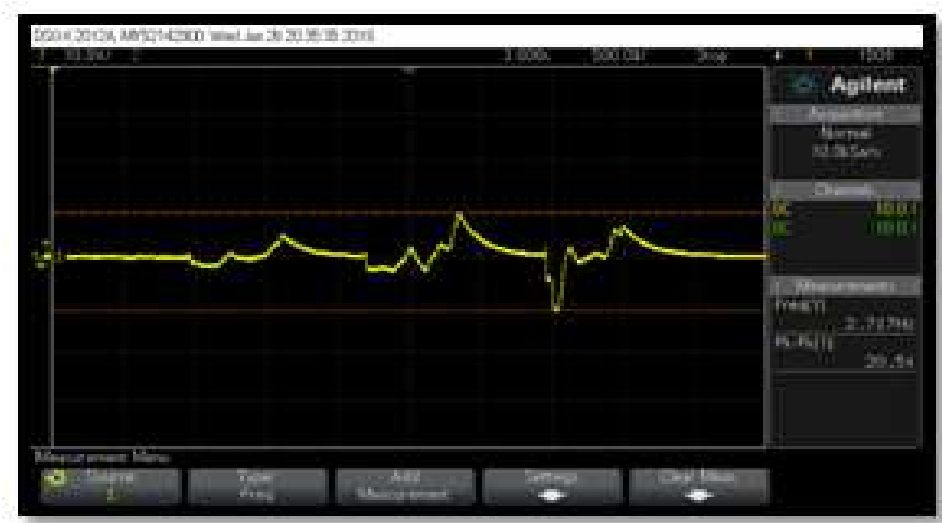

Figure 11: Index Finger Pressure Recording

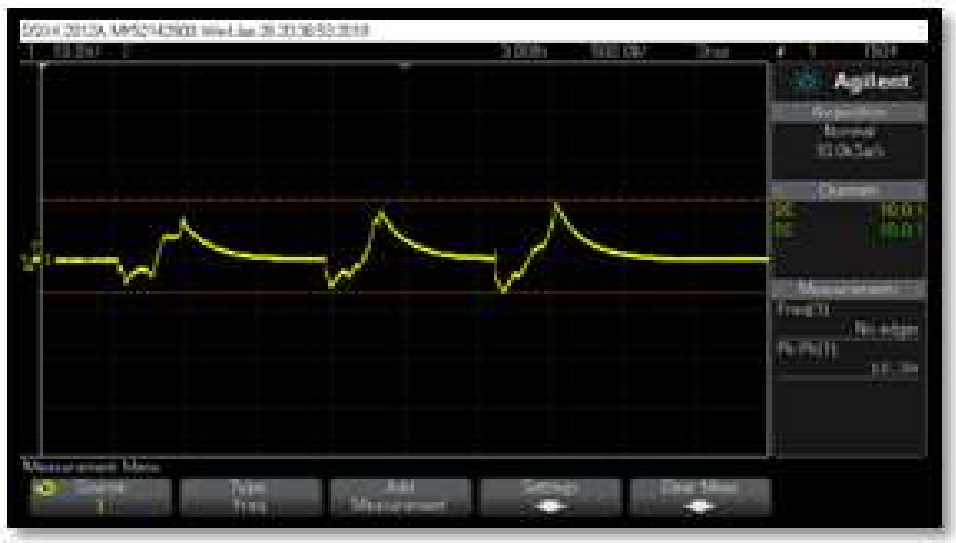

Figure 12: Middle Finger Pressure Recording

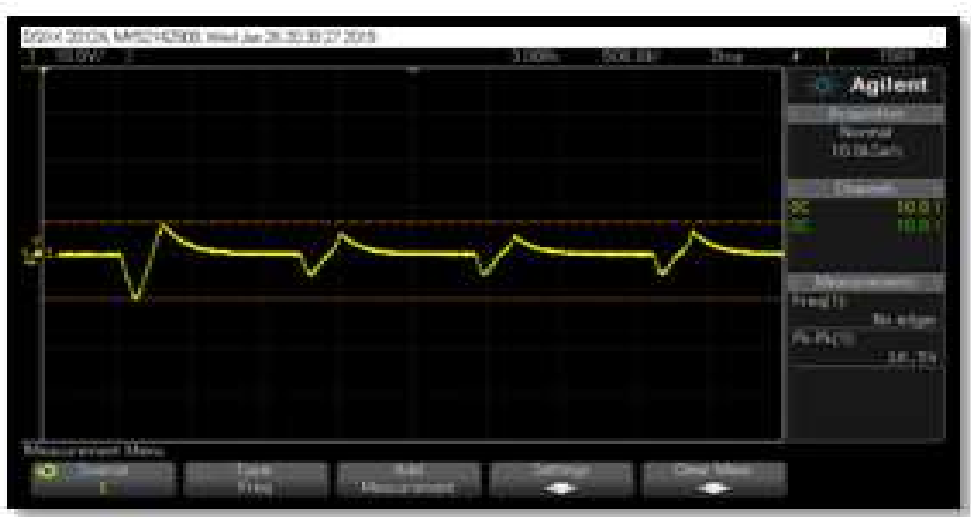

Figure 13: Ring Finger Pressure Recording 


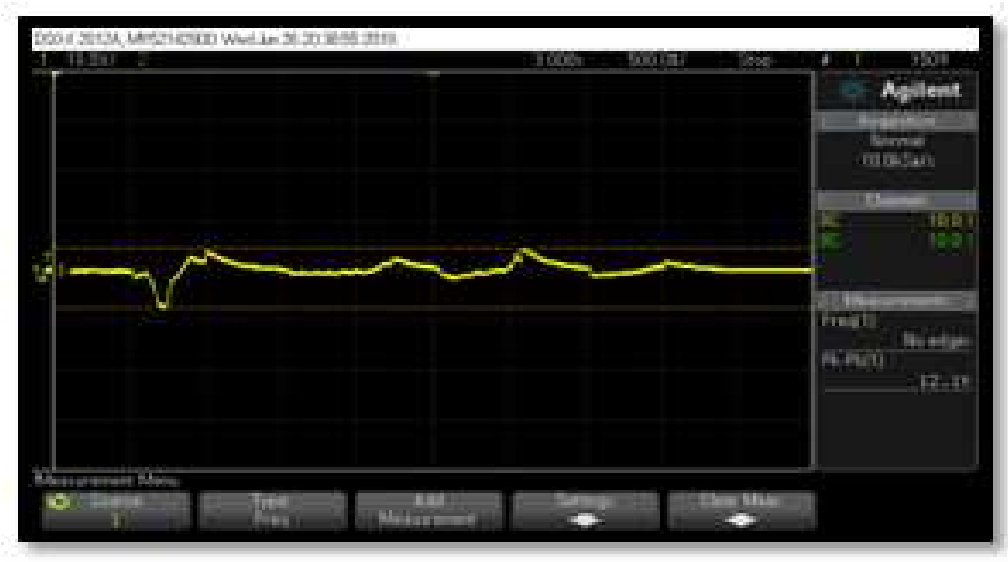

Figure 14: Little Finger Pressure Recording

The EMG along with the force-touch sensor portrays the pressure element by sending the signal from the pressure sensor to EMG or vice versa if pressure is applied by the hand, and to vibrator module if the pressure is applied on the hand.

\section{Temperature Sensor Explanation}

The thermistor works along with the Peltier module, as mentioned above in need of sensory feedback. The temperature of Peltier module changes depending on the heat or cold sensed near the tip of the fingers. This value is then sent to the microcontroller, which compares with the threshold value, and when it crosses the value, the withdrawal-mechanism takes place.

\section{HARDWARE SIMULATION RESULTS}

The board-mounted sensors work with the specifications as mentioned below:

\begin{tabular}{|c|l|l|}
\hline S. No. & \multicolumn{1}{|c|}{ Specifications } & \multicolumn{1}{|c|}{ range } \\
\hline $\mathbf{1}$ & Operating Pressure & $7 \mathrm{psi}$ \\
\hline $\mathbf{2}$ & Pressure Type & Absolute \\
\hline $\mathbf{3}$ & Operating Supply Voltage & $10 \mathrm{~V}$ \\
\hline $\mathbf{4}$ & Minimum Operating Temperature: & $-40 \mathrm{C}$ \\
\hline $\mathbf{5}$ & Maximum Operating Temperature: & $+125 \mathrm{C}$ \\
\hline $\mathbf{6}$ & Operating Supply Current & $6 \mathrm{~mA}$ \\
\hline 7 & Grasp speed & $1.5-2$ seconds \\
\hline
\end{tabular}

Figure 15: Specifications of the Board-Mounted Sensors

The table 15 displays the desired output characteristics to a very reasonable extent to exhibit the arm in the market for use.

\section{CONCLUSIONS}

The proposed prosthetic hand model with a sensory feedback system has shown its fair ability to qualify as an efficiently functioning hand for an amputee. Due to various elements present in the designed prosthetic hand, having the EMG, touch, 
and temperature sensors make the user manipulate a wide variety of objects ranging from a thin foil to a moderately heavy object useful in ADLs.

With potent operating sensors, the user has less risk of moving a hot object near/to his mouth as the temperature sensor with Peltier module has that result ready and functioning accordingly; the EMG along with touch/pressure sensor would analyze the risk of overweight. Although there can be a discussion about increasing the grasp speed and higher degrees of freedom along with weight-bearing capacity ultimately, the proposed hand with sensory feedback has a human hand shape, size, and sensation to a great extent.

\section{REFERENCES}

1. J. T. Belter, J. L. Segil, A. M. Dollar, and R. F. Weir, "Mechanical design and performance specifications of anthropomorphic prosthetic hands: A review," J. Rehabil. Res. Develop., vol. 50, no. 5, pp. 599-618, 2013.

2. Sheila, T. "Marketing of Paddy-A Study with Special Reference to Alanganallur Block, Madurai District." International Journal of Agricultural Science and Research (IJASR) 6.3 (2016).

3. Myoelectric prosthetic hand with a proprioceptive feedback system, science direct.

4. Guidelines for Training Personnel in Developing Countries for Prosthetics and Orthotics Services, World Health Organization, Geneva, Switzerland, 2005.

5. Yuvarani, G., et al. "To compare the effectiveness of Laser, EMG biofeedback assisted core stability exercise versus Laser and Core stability exercise alone on pain and disability in patients with non-specific low back pain." Research Journal of Pharmacy and Technology 13.6 (2020): 2563-2566.

6. WHO Standards for Prosthetics and Orthotics, World Health Organization, Geneva, Switzerland, 2017.

7. Deepan, V., M. Subramanian, and C. Dineshkumar. "Motorcycle rider fatigue analyze: Results of an online survey." Int. J. Mech. and Production, Engg., Research and Development 8.2 (2018): 509-516.

8. World Population by Region-[Online, 2017]. Available: http://www. worldometers.info/world-population/\#region.

9. Exploring Human Hand Capabilities into Embedded Multifingered Object Manipulation, IEEE Transactions on Industrial Informatics, Vol. 7, No. 3, August 2011 

